\title{
TinyNode: A Comprehensive Platform for Wireless Sensor Network Applications
}

\author{
Henri Dubois-Ferrière \\ EPFL \\ 1015 Lausanne \\ Switzerland \\ henri.dubois-ferriere@epfl.ch
}

\author{
Roger Meier \\ Shockfish SA \\ 1015 Lausanne \\ Switzerland \\ roger@shockfish.com
}

\author{
Laurent Fabre \\ EPFL \\ 1015 Lausanne \\ Switzerland \\ laurent.fabre@epfl.ch
}

\author{
Pierre Metrailler \\ Shockfish SA \\ 1015 Lausanne \\ Switzerland \\ metrailler@shockfish.com
}

\begin{abstract}
We introduce the TinyNode platform for wireless sensor networks. Supporting both research and industrial deployments, the platform offers communication ranges that exceed current platforms by a factor of 3 to 5 , while consuming similar energy. It comes with a rich, practical set of hardware extensions and full TinyOS support. We describe the design choices of the TinyNode, the accompanying hardware modules, and the MAC layer implementation.
\end{abstract}

Categories and Subject Descriptors: C.2.1 [ComputerCommunications Networks]: Wireless Communication

General Terms: Measurement, Performance, Design, Experimentation.

Keywords: Wireless Sensor Networks, Embedded Systems.

\section{INTRODUCTION}

Wireless sensor networks are emerging as an enabling technology for many applications which require as input quantities measured at multiple points in the physical world. However the requirements of different applications can vary widely in almost every aspect. In terms of hardware, a node for environmental or habitat monitoring [1] [2] will be placed outdoor and have multiple atmospheric sensors, whereas a node for embedded industrial applications (such as machine monitoring, [3]) may employ only a single vibration sensor but have stringent space requirements. A node for a research testbed is typically indoor, does not sense real phenomena, and requires a wired backchannel for control and measurements. In most deployments, a few basestation or clusterhead nodes require a longer haul, higher capacity connection such as WLAN or GSM. Energy requirements are also different, ranging from ultra-low duty cycle applications, which may survive on small batteries for several years, to higher duty cycle applications which require energy harvesting (e.g., of solar power) for autonomous long-term operation. Finally communication ranges may go from meters for highly dense deployments (e.g., microclimate monitoring

Permission to make digital or hard copies of all or part of this work for personal or classroom use is granted without fee provided that copies are not made or distributed for profit or commercial advantage and that copies bear this notice and the full citation on the first page. To copy otherwise, to republish, to post on servers or to redistribute to lists, requires prior specific permission and/or a fee.

IPSN'06, April 19-21, 2006, Nashville, Tennessee, USA

Copyright 2006 ACM 1-59593-334-4/06/0004 ...\$5.00.
[4]) to hundreds of meters for sparse networks covering large areas.

In this paper, we introduce the design of the TinyNode platform and its accompanying hardware extensions. The design philosophy of TinyNode is to place core components which are required for every application on a small module, and additional functionality on extension boards. The core module is a versatile low-power wireless node, and comes with an array of extension hardware offering a wide set of connectivity, storage, energy, and interfacing options. It uses a low power transceiver which has energy characteristics comparable to those found on other sensor nodes ("motes"), but offers a significantly larger range, and bit rates from $1.2 \mathrm{kbps}$ all the way up to $152 \mathrm{kbps}$. The platform comes with full TinyOS support, including a complete radio stack, support for network reprogramming with Deluge, [5] and bridging software for GPRS/GSM data transfer.

\section{PLATFORM ARCHITECTURE}

The architecture of the TinyNode platform suite is organized around a core module (Fig. 1), and optional peripherals which can be selected based on application needs.

\subsection{TinyNode Core Module}

Fig. 2 shows a block diagram of the core module, which contains the strict minimum common components required for operation: microcontroller, radio, flash, voltage regulator and supply monitor, and an expansion connector.

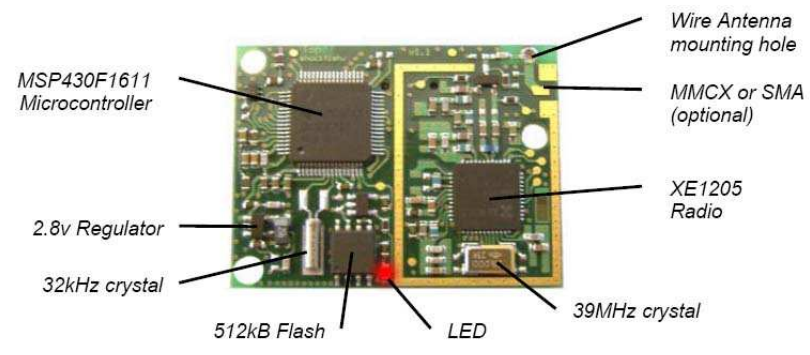

Figure 1: TinyNode Core module (upper sides).

\subsubsection{MSP430 Microcontroller}

The TinyNode features a MSP430F1611 ultra-low power microcontroller that is fully supported by TinyOS and has the lowest power consumptions and fastest wake-up cycles available today. The digitally controlled oscillator (DCO) allows 
wake-up from low-power modes to active mode in less than $6 \mu \mathrm{s}$ and may operate up to $8 \mathrm{MHz}$. Typically, the DCO will turn on from sleep mode in 300ns at room temperature. The MSP430F1611 has two built-in 16-bit timers, a fast 12-bit A/D converter, dual 12-bit D/A converters, one or two universal serial synchronous/asynchronous communication interfaces (USART), I2C, DMA, and $48 \mathrm{I} / \mathrm{O}$ pins. The same microcontroller is used on the Telos [6] and EyesIFX [7] platforms. We refer to [6] for a full comparison of the MSP430F1611 with competing microcontrollers from Atmel, Motorola, and Microchip. The core module also has a 4Mbit flash chip that can be used for storing several firmware images or for logging data.

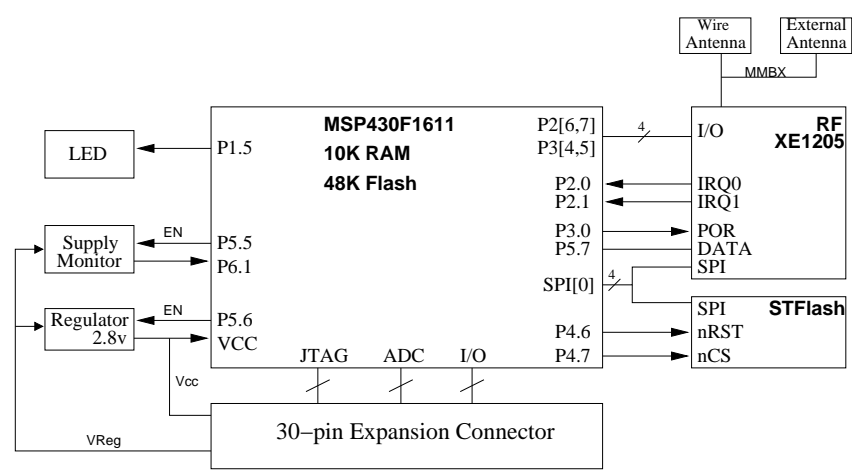

Figure 2: TinyNode core module block diagram.

\subsubsection{XE1205 Radio}

Particular attention has been put to the choice of the radio transceiver. The XE1205 from Semtech (formerly XEMICS) is an integrated transceiver that can operate in the 433,868 and $915 \mathrm{MHz}$ license-free ISM frequency bands. The version used for the measurements given in this paper operates at 868Mhz.

All major RF communication parameters are programmable and most of them can be dynamically set. The XE1205 offers both narrow-band and wide-band communication with the same hardware configuration, allowing data rates from $1.2 \mathrm{kbit} / \mathrm{s}$ to $152 \mathrm{kbit} / \mathrm{s}$.

Compared to other transceivers in the market (including Chipcon, Nordic, RFM, Micrel, TI, Infineon), the XE1205 offers the highest link budgets available today in the license free ISM bands. With an output power of $+15 \mathrm{dBm}$ and sensitivity of $-116 \mathrm{dBm}$ at $4.8 \mathrm{kbit} / \mathrm{s}$, a link budget of $131 \mathrm{~dB}$ can be achieved. This is around $22 \mathrm{~dB}$ better than for the Chipcon CC1000 radio used on the Mica2 platform, which gives the TinyNode around 4 times longer range.

Table 1 shows the key transceiver characteristics for the CC1000, CC2420, TDA5250, and XE1205 radio transceivers. The link budget is the sum of all signal gains and losses over the entire wireless path, and the receiver sensitivity is the signal level at which the decoded signal has a bit error rate (BER) below 0.1\%. For comparison, the antenna gain is assumed to be unitary $(0 \mathrm{dBi})$ for all platforms and the outdoor range is calculated according to an isotropic path loss model with a gain exponent of $n=2.6$ for open field propagation.

For low data rates, the RF frequency needs to be controlled carefully. The built-in frequency error indicator (FEI) of the XE1205 allows implementing an automatic frequency control loop (AFC) by software, avoiding the need for an expensive temperature compensated oscillator.

In addition to excellent range performance, the XE1205 has a modern zero-IF architecture that offers several advantages over traditional architectures that use one or more intermediate frequencies (as used in the the CC1000, CC2420, or TDA5250 transceivers). With zero-IF, the analog RF signal is directly converted to a digital baseband signal. Such architecture limits the number of required active blocks and avoids cumbersome external IF band pass or SAW filters for channel selection. Moreover, the baseband signal does not need to be DC balanced and can be NRZ coded. Compared to a Manchester encoded bit stream (typically used for Mica2 nodes), the effective data rate is double.

The internal 16 Byte FIFO buffer and the automatic pattern detector of the XE1205 reduce CPU load during time-critical transmit and receive loops. Using these features, we were able to run the radio at full $152 \mathrm{kbit} / \mathrm{s}$ under TinyOS.

\subsubsection{Energy consumption.}

Energy consumption is a critical parameter of a sensor node. Table 2 shows the current consumption for Mica2, Telos, EyesIFX, and TinyNode. The MCU related consumptions of the TinyNode, EyesIFX, and Telos nodes are identical since they use the same chip. TinyNode has radio consumptions comparable to the Mica2, while offering significantly higher range and data rates. Telos also has comparable radio consumption, but the CC2420 offers a higher bit rate and faster radio wake-up. The tradeoff to this lower consumption is a reduced communication range.

\subsection{Extension Boards}

\subsubsection{Standard Extension Board}

The Standard Extension Board (SEB) is designed as a lowcost extension to the TinyNode and provides the necessary functionality for development purposes, simple deployments and hardware prototyping. It is a strict subset of the MamaBoard; in other words all of the hardware and functionality of the SEB is also present in the MamaBoard.

The SEB can be powered either from battery or from an external supply through a Jack connector. For programming and debugging, it includes a JTAG interface and a RS-232 with full BSL support. The RS-232 drivers are powered directly from the serial line, avoiding drawing current from the battery.

The board includes footprints for two optional sensors: a relative humidity and temperature sensor (Sensirion SHT11), and a photodiode light sensor (Infineon BPW345-P1602). Elements for basic interaction with a running system are 3 light-emitting diodes (LEDs) and 4 jumpers that can be read on the microcontroller's digital inputs.

The SEB also offers a pad field that can be used to quickly connect custom sensors or actuators. The pad field has a row of unused pins coming from the microcontroller on the one side and a row of pads that are connected to a standard flat cable connector on the other side. In the middle, there are unused pin-through-hole pads that can be used to solder simple glue electronics.

\subsubsection{MamaBoard}

In addition to the functionality of the SEB, the MamaBoard offers rich connectivity and storage options, allowing to 


\begin{tabular}{|l|c|c|c|c|c|c|c|}
\hline Platform & \multicolumn{2}{|c|}{ Mica2 } & Telos Sky & \multicolumn{2}{c|}{ EyesIFX } & \multicolumn{2}{c|}{ TinyNode } \\
\hline Transceiver & \multicolumn{2}{|c|}{ CC1000 } & CC2420 & \multicolumn{2}{|c|}{ TDA5250 } & \multicolumn{2}{|c|}{ XE1205 } \\
Frequency & \multicolumn{2}{|c|}{$869 \mathrm{Mhz}$} & $2.4 \mathrm{Ghz}$ & \multicolumn{2}{|c|}{$869 \mathrm{Mhz}$} & \multicolumn{2}{|c|}{$869 \mathrm{Mhz}$} \\
Max. Tx Power & \multicolumn{2}{|c|}{$5 \mathrm{dBm}$} & $0 \mathrm{dBm}$ & \multicolumn{2}{|c|}{$9 \mathrm{dBm}$} & \multicolumn{2}{c|}{$15 \mathrm{dBm}$} \\
\hline Data Rate & $76.8 \mathrm{kbps}$ & $4.8 \mathrm{kbps}$ & $250 \mathrm{kbps}$ & $64 \mathrm{kbps}$ & $4.8 \mathrm{kbps}$ & $76.8 \mathrm{kbps}$ & $4.8 \mathrm{kbps}$ \\
Sensitivity & $-98 \mathrm{dBm}$ & $-104 \mathrm{dBm}$ & $-94 \mathrm{dBm}$ & $-96 \mathrm{dBm}$ & $-110 \mathrm{dBm}$ & $-106 \mathrm{dBm}$ & $-116 \mathrm{dBm}$ \\
Link Budget & $103 \mathrm{~dB}$ & $109 \mathrm{~dB}$ & $94 \mathrm{~dB}$ & $105 \mathrm{~dB}$ & $119 \mathrm{~dB}$ & $121 \mathrm{~dB}$ & $131 \mathrm{~dB}$ \\
Range Outdoor $^{1}$ & $160 \mathrm{~m}$ & $300 \mathrm{~m}$ & $80 \mathrm{~m}$ & $200 \mathrm{~m}$ & $600 \mathrm{~m}$ & $600 \mathrm{~m}$ & $1800 \mathrm{~m}$ \\
\hline
\end{tabular}

Table 1: Comparison of radio transceiver characteristics.

\begin{tabular}{|l|c|c|c|c|c|}
\hline & Mica2 & Telos Sky & EyesIFX & TinyNode & \\
\hline Min Voltage & 2.7 & 1.8 & 2.1 & 2.4 & $\mathrm{~V}$ \\
Max Voltage & 3.3 & 3.6 & 3.6 & 3.6 & $\mathrm{~V}$ \\
MCU sleep with RTC on (LPM3) & 19 & 5.1 & 5.1 & 5.1 & $\mu \mathrm{A}$ \\
MCU active & 8 & 1.8 & 1.8 & 1.8 & $\mathrm{~mA}$ \\
MCU active, Radio RX & 15.1 & 21.8 & 10.8 & 15.8 & $\mathrm{~mA}$ \\
MCU active, Radio TX at +0dBm (1mW) & 25.4 & 19.5 & 13.7 & 25 & $\mathrm{~mA}$ \\
MCU active, Flash Read & 9.4 & 4.1 & 5 & 5 & $\mathrm{~mA}$ \\
MCU active, Flash Write & 21.6 & 15.1 & 16 & 16 & $\mathrm{~mA}$ \\
MCU wake-up latency & 180 & 6 & 6 & 6 & $\mu \mathrm{s}$ \\
Radio wake-up latency & 1800 & 580 & 2200 & 1500 & $\mu \mathrm{s}$ \\
\hline
\end{tabular}

Table 2: Current consumption and wake-up times.

bridge a wireless sensor network to wired ethernet (LAN), WLAN, or cellular GPRS. These three connectivity types are achieved by plugging optional external modules onto the board. For robust and low-cost mass storage, the board includes a SD memory card slot which can be accessed both by the TinyNode and by the GPRS module.

\section{LAN and WLAN connectivity}

LAN connectivity is achieved by plugging a Digi Connect ME [8] and WLAN connectivity with a Digi Connect WiME. Both modules are IP-capable devices that offer transparent serial port relaying over TCP/IP, allowing a host PC to "mount" the serial port over the network and interact with the remote TinyNode as if it were locally connected to the PC's serial interface. Using the wired ethernet option, a testbed of TinyNodes with wired backchannel can be set up with little effort and at low cost (the Digi Connect ME can be purchased for approximately $\$ 50)$. We have recently installed a 50-node testbed at EPFL using the Digi Connect $\mathrm{ME}$ as a wired backchannel. The Digi Connect Wi-ME offers the same functionality as the ME, but over WLAN.

\section{GPRS/GSM Connectivity}

In addition to Ethernet and WLAN, the third connectivity option provided by the MamaBoard is GPRS. GPRS is a packet-switched mobile data service available in GSM cellular networks. With GSM/GPRS connectivity, we aim for the following requirements: (i) sending measurements received on the MamaBoard-hosted TinyNode to a central server over the Internet, (ii) remotely controlling the MamaBoard by SMS, and (iii) performing software updates of all nodes attached to the wireless sensor network reachable from the MamaBoard.

SD Memory Card MamaBoard also provides a Secure Digital (SD) card slot for mass storage. SD is a flash memory card format that has become widely used in portable devices. Typical capacities today are 128, 256 and 512 megabytes, 1, 2 and 4 gigabytes. Interfacing with the TinyNode is straightforward, since all SD memory cards are required to support the older SPI/MMC serial mode that is compatible with the MSP430 SPI ports. The SD card can be used as a robust and low-cost mass storage device for data logging applications or as a temporary buffer, in case the connectivity to the MamaBoard is lost. It can be accessed both by the TinyNode and the GPRS module.

\section{XE1205 TINYOS RADIO STACK}

The port of TinyOS to the TinyNode platform consists essentially of low-level hardware adaptation code and a new radio driver and MAC layer for the XE1205 transceiver. The hardware adaptation phase made full use of the Hardware Abstraction Architecture (HAA) already developed at UC Berkeley and TU Berlin [9] with support for the MSP430 microcontroller. Our experience with this HAA has been very positive.

Unlike the core platform support, the radio stack had to be written from scratch, since the XE1205 transceiver has not been previously used in TinyOS-supported platform. We have designed and implemented a full radio stack around the XE1205 which includes CSMA, acknowledgement frames, low-power listening, and support for bit rates all the way up to $152 \mathrm{kbps}$. The radio stack is relatively compact (206 bytes RAM and 6126 bytes ROM, including HPL and BusArbitration code).

The XE1205 interfaces to the microcontroller using SPI. It offers a bytewise read/write interface for sending and receiving data, and is configured with register operations over SPI. Support for full-speed operation at $152 \mathrm{kbps}$ would be difficult with a bare bytewise interface (such as that of the Chipcon CC1000), since every single byte (transmitted or received) must be handled in less than $50 \mu \mathrm{s}$. As a comparison, the TinyOS driver for the Mica2's CC1000 transceiver operates at $19.2 \mathrm{kbps}$, giving it up to $416 \mu \mathrm{s}$ to handle each byte.

Fortunately, the XE1205 includes some functionality which helps to offload the microcontroller. In particular, it offers a 


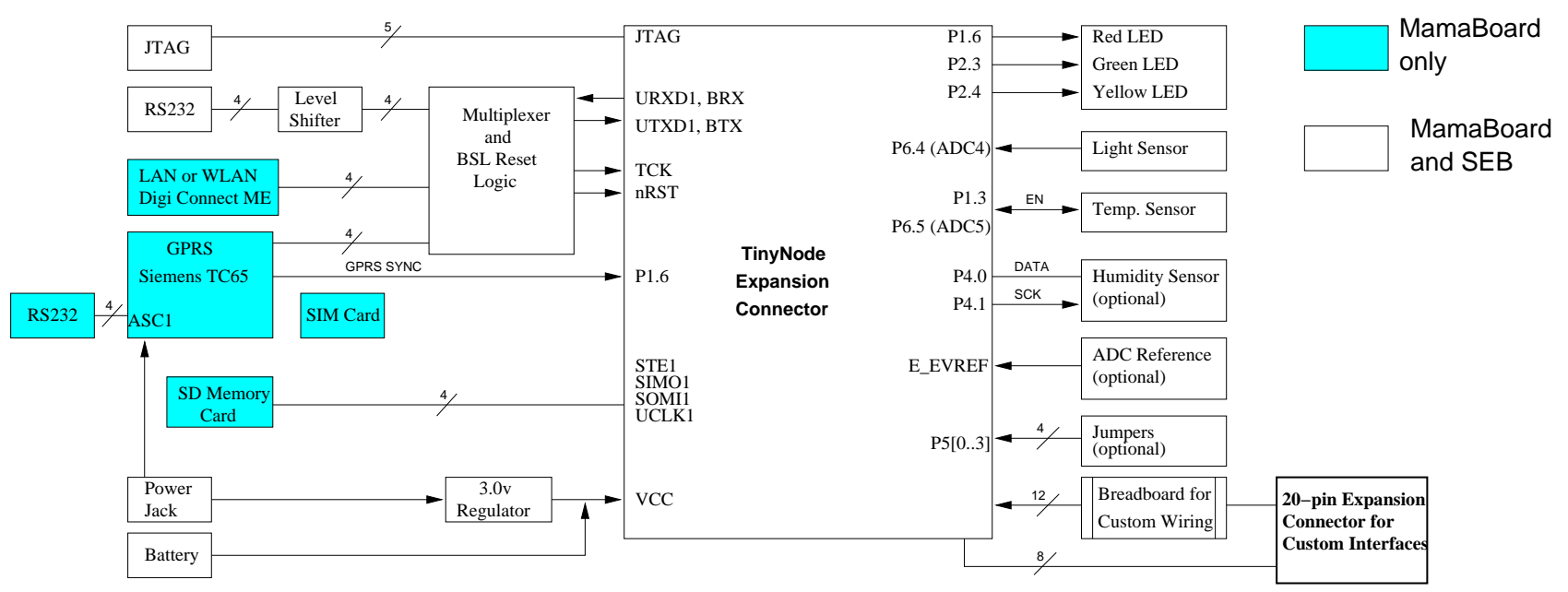

Figure 3: SEB/MamaBoard block diagram.

16-byte FIFO buffer for sending and receiving packets, and a hardware preamble detector which generates an interrupt as soon as a configurable preamble (of length 8 to 32 bits) is received. While the FIFO buffer avoids having to read (write) every byte as it arrives (transmits), a latency below $50 \mu \mathrm{s}$ is still necessary during packet reception of long packets each time the FIFO reaches 16 bytes, and during transmission each time the FIFO's becomes empty. At this point, the driver must respond rapidly enough to read (write) 16 bytes from (into) the FIFO, otherwise an incoming byte will be lost, or the outgoing bitstream will contain a gap and lose synchronisation.

To evaluate software overhead, we measured processing and switching times by sending a continuous packet stream, with initial backoffs disabled, and computing the total channel utilization. While this is not a realistic application profile, it allows us to evaluate if there is any inefficiency in the packet-processing and switching times. Results show that the driver is fast: total channel utilization when sending a continuous packet stream, is $68.8 \%$ at $152 \mathrm{kbps}, 80.2 \%$ at $76 \mathrm{kbps}$ and $94.7 \%$ at $19.2 \mathrm{kbps}$. In comparison, the Mica2 stack running at $19.2 \mathrm{kbps}$ has approximately $85 \%$ channel utilization in the same conditions. For TinyNode, utilization decreases with bit rate, because the per-packet overhead has a constant component which is independent of bit rate.

\subsection{Low Power Listening Implementation}

For duty cycling, the XE1205 radio stack implements a technique known as low power listening (or preamble sampling) [10] [11]. Low power listening achieves a low duty cycle by having nodes periodically awaken for short periods and listen on the radio. If a node detects an ongoing packet preamble transmission, it remains awake to receive the packet; otherwise it returns to sleep until the next wakeup time. A transmitter sends a packet with a preamble of length sufficient to cover the receiver sleep period. This technique has the advantage of being simple and robust and does not require node synchronization or any other form of coordination between neighboring nodes.

The implementation takes advantage of the XE1205 pattern detector: when a node wakes up, it programs the pattern detector with a two-byte pattern 1010101.., corresponding to the preamble sequence. If these bytes are received, the radio signals an interrupt, and the node now knows that (with high probability) a packet preamble is ongoing. It then reprograms the preamble detector with a 3-byte start-of-frame sequence, and awaits a second interrupt signalling the start of packet reception. This use of the preamble detector allows to significantly off-load the microcontroller in comparison to a software implementation which must process every byte of the preamble, as with the CC1000.

For real performance, it is interesting to examine a typical low power listening (LPL) mode with $1 \%$ duty cycle, meaning that the receiver is active during $1 \%$ of the time for listening. At $152 \mathrm{kbit} / \mathrm{s}$, we obtain a minimum listen period of $1.9 \mathrm{~ms}$ (including radio start-up time and RSSI measurement), which means the listening period is $190 \mathrm{~ms}$ for a $1 \%$ activity. In comparison, due to higher start-up times and lower data rates, a Mica2 node at $19.2 \mathrm{kbit} / \mathrm{s}$ has $8 \mathrm{~ms}$ of listening time and a listening interval of approximately 1 second. In comparison, this represents a fivefold improvement in latency (or equivalently, throughput) over Mica2, with comparable battery lifetime and range. Conversely, if an application can tolerate a 1-second per-hop latency but requires minimizing energy consumption, the TinyNode can run at $0.2 \%$ duty cycle and consume almost an order of magnitude less than Mica2, whilst offering the same delay as the Mica2 at $1 \%$ duty cycle. In this case, a theoretical lifetime of over 6 years can be achieved with $2 \times$ AA alkaline cells. Note that the relative improvement is smaller than the ratio of bit rates, because both listen times include a radio wake-up time. While the XE1205 wake-up time is shorter than the CC1000 wakeup time, its relative duration when counted in byte times at $152 \mathrm{kbps}$ is higher than for the CC1000. Table 3 summarizes these numbers. Note that due to self-discharge and degradation, another type of battery technology needs to be used to obtain such lifetimes (such as Lithium Thionyl Chloride).

\subsection{Radio Range}

The hardware characteristics of the XE1205 transceiver shown in Table 1 indicate a theoretical range of up to $1800 \mathrm{~m}$ 


\begin{tabular}{|c|c|c|c|}
\hline & Mica2 at $1 \%$ & TinyNode at $1 \%$ & TinyNode at $0.2 \%$ \\
\hline Bit Rate & $19.2 \mathrm{kbps}$ & $152 \mathrm{kbps}$ & $152 \mathrm{kbps}$ \\
\hline Listen Time & $8 \mathrm{~ms}$ & $1.9 \mathrm{~ms}$ & $1.9 \mathrm{~ms}$ \\
\hline Listen Period (Max. Latency) & $1085 \mathrm{~ms}$ & $190 \mathrm{~ms}$ & $950 \mathrm{~ms}$ \\
\hline Max throughput & $0.89 \mathrm{pkts} / \mathrm{sec}$ & $5.5 \mathrm{pkts} / \mathrm{sec}$ & $1.05 \mathrm{pkts} / \mathrm{sec}$ \\
\hline Average Power Consumption & $509 \mu \mathrm{W}$ & $489 \mu \mathrm{W}$ & $104 \mu \mathrm{W}$ \\
\hline Lifetime $^{2}$ for $2 \times$ AA alkaline cells, $2000 \mathrm{mAh}$ & 1.3 years & 1.4 years & 6.6 years \\
\hline
\end{tabular}

Table 3: Duty-cycled power consumption.
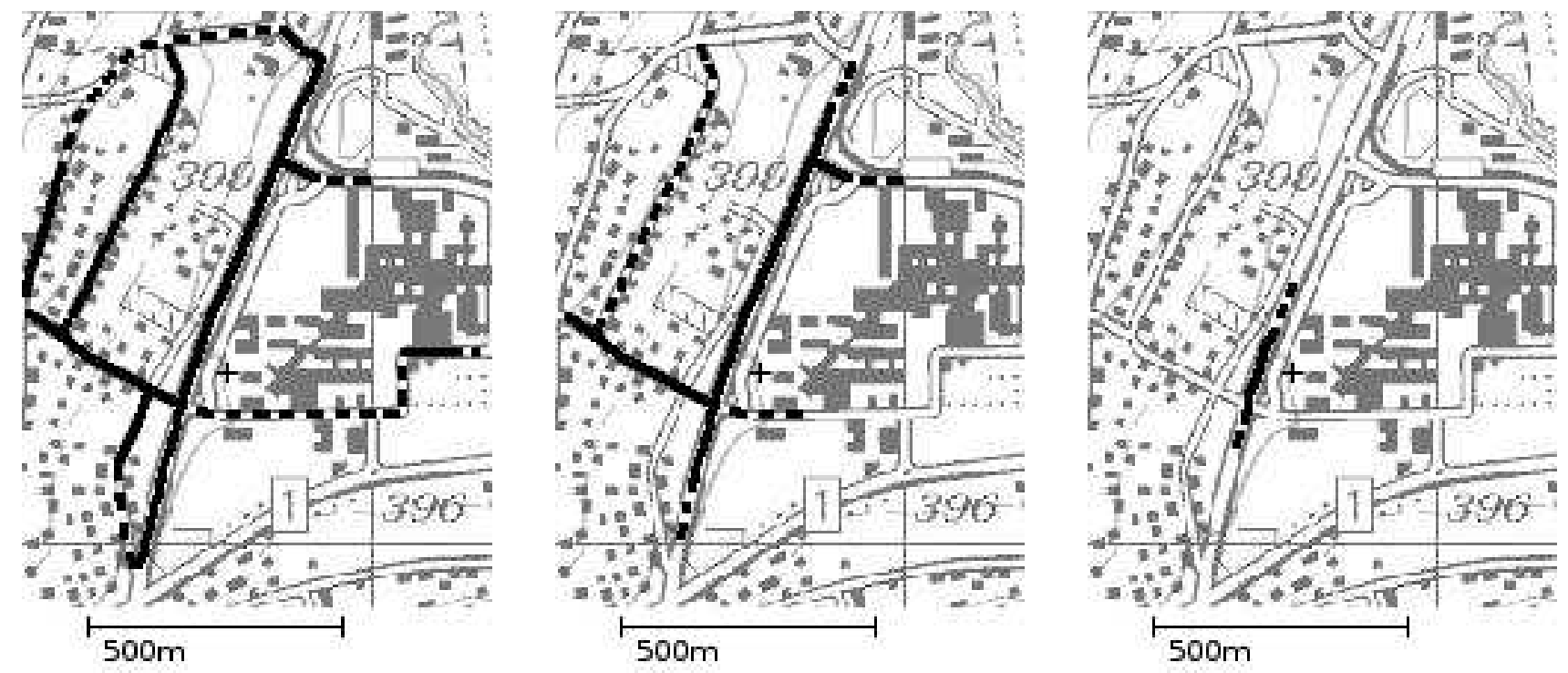

Figure 4: Range traces with TinyNode and Telos transmitting TinyOS packets. Left: TinyNode at 76 kbps, 15dBm transmit power. Center: TinyNode at $76 \mathrm{kbps}, 0 \mathrm{dBm}$ transmit power. Right: Telos at 250kbps, 0dBm transmit power. The transmitter is marked with a cross, near the South West side of campus. A solid line indicates a reliable link from a node in a moving car to the eceiver in the car, and a dotted line indicates areas of unreliable reception

at low bit rates. While link budget, sensitivity, and theoretical ranges allow for high-level comparison between different transceivers, it is necessary to validate them with empirical measurements in order to ascertain that whole system performance is in line with expectations.

We ran some simple experiments in order to show the ranges achievable with TinyNode and Telos nodes. We placed a transmitter on the balcony of a 4-floor building and a receiver on the roof of a car. Nodes were mounted with $1 / 4$ wave monopole antennas with approximately $0 \mathrm{dBi}$ of gain [12] [13]. We then drove a circuit with the car while observing the connection. The area of this experiment is a hilly (elevations are shown on the map in italicized font) urban and suburban area. During most of the circuit, we were not in line of sight of the transceiver. The only line-of-sight segment is a $200 \mathrm{~m}$ portion of the road in the immediate vicinity of the transmitter.

In the first run, we compared the TinyNode and Telos ranges at high bitrates $\left(76 \mathrm{kbps}\right.$ and $250 \mathrm{kbps}$ respectively ${ }^{3}$ ), with nodes running the standard TinyOS CntToRfm and RfmToLeds applications. The traces are shown in Fig. 4.

In the second run, we aimed to push the TinyNode range to its limits. The bit rate was set to $4.8 \mathrm{kbps}$, and nodes sent short (6 byte) frames. The receiver also ran an automatic

\footnotetext{
${ }^{3}$ The CC2420 radio only supports this single bitrate.
}

frequency control loop, driven by the frequency error indicator of the XE1205. The trace of this experiment is shown in Fig. 5. We were able to communicate up to $2.3 \mathrm{~km}$ (North segment near 'Crochy') without direct line-of-sight.

These experiments confirm (and exceed) the theoretical outdoor range of Table 1 . This outdoor range is higher than that of widespread platforms (such as Mica2 or Telos) by a factor of 4 to 8 . With such a radio range, TinyNode enables wide-area sensing applications that were previously not possible. Even assuming a conservative average range estimate of $250 \mathrm{~m}$, a sizeable urban area can be covered without requiring thousands of nodes. For example, a city such as San Francisco (approximately $8 \mathrm{~km}$ by $8 \mathrm{~km}$ ) could be covered with approximately 1000 nodes, whereas with a radio range of $80 \mathrm{~m}$ this would require over 10000 nodes.

\section{SOLAR SUPPLY}

We have also designed a solar energy supply board for longterm outdoor operation. This system operates with a small solar panel, a primary energy buffer, and optionally a secondary energy buffer. Our first goal in the design of this system is flexibility of hardware configurations, in order to meet widely varying energy budgets of different applications. The overall architecture is similar to that of Prometheus [14]: a primary buffer is designed to collect energy from the 


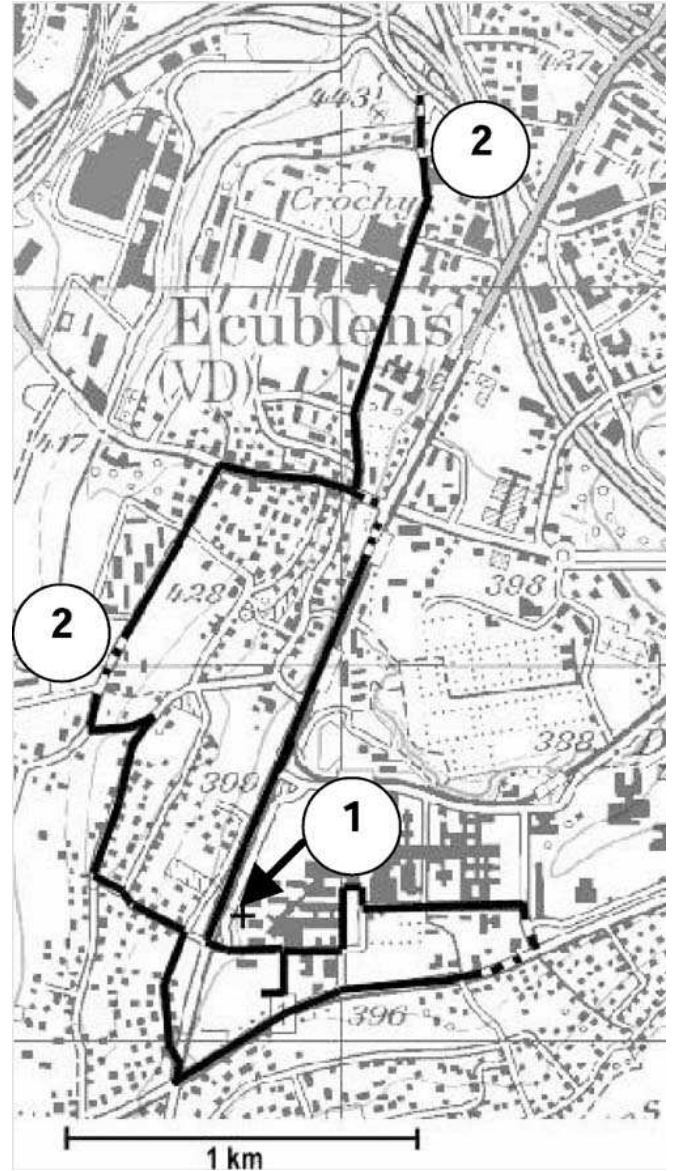

Figure 5: Range trace with TinyNode transmitting short frames at $4.8 \mathrm{kbps}$, with automatic frequency control, $15 \mathrm{dBm}$ transmit power. (1): Transmitter position. (2): Areas of unreliable reception.

solar panel, powering the node whenever possible, and an optional secondary buffer, with higher capacity, is used for extended operation when solar energy is not sufficient to fill the primary buffer. Unlike Prometheus, our aim is not for perpetual or near-perpetual unattended operation. ${ }^{4}$ However, the tiered buffer strategy remains valuable to relieve the secondary buffer of excessive cycles, and to have a source of backup energy during long periods of low solar energy. Our second goal is to offer complete control and monitoring interfaces to the attached node, in order to allow the implementation in software of energy management algorithms adapted to the application requirements. Specifically, the node can measure the following quantities: primary buffer voltage $U_{\text {prim }}$, secondary buffer voltage $U_{\text {sec }}$, external power supply voltage $U_{\text {ext }}$, the solar panel current $I_{\text {solar }}$, the charge current of the primary buffer $I_{\text {charge }}$, and the overall current consumption of the TinyNode and any additional sensors $I_{\text {supply }}$. We use two ADC ports on the MSP430 microcontroller for these measures: one for voltages, and one for currents. We use a current monitor (Zetex

\footnotetext{
${ }^{4}$ To our best knowledge, all batteries available today degrade and lose their capacity over time, even without going through charging cycles.
}

ZXCT1010) to measure currents. The input on either ADC port is selected using two multiplexers which are controlled with two digital outputs from the MSP430.

\subsection{Choice of components}

The key system components are the solar panel, primary buffer, and secondary buffer. Our criterion for the solar panel was that it should generate sufficient power to charge the primary buffer in less than a day. This should hold even in poor luminosity conditions, in order for the energy subsystem to work in short winter days. We settled on a 140x40mm solar panel with has a nominal power output of $300 \mathrm{~mW}$ in direct sunlight. The role of the secondary buffer is to offer an energy backup source that can allow the node to operate for periods when the solar panel is not providing sufficient power to drive the primary buffer. The length of such black-out periods depends essentially on weather factors and on the geographic location of a deployment. We are currently using a Li-Ion battery [15] from Leclanché which has a capacity of $2000 \mathrm{mAh}$ under 3.7 Volts.

The final component to choose is the primary buffer. We selected three options covering a large capacity range. These are summarized in Table 4. Each option is matched to different requirements. The first option has lowest capacity. It uses two $1 \mathrm{~F}$ supercapacitators [16] which are wired in series in order to obtain an operating voltage of up to $5.5 \mathrm{~V}$. This gives a total capacitance of $0.5 \mathrm{~F}$. It has lowest cost and is matched to a system where good solar exposure can be expected on a near-daily basis, allowing the node to charge the secondary buffer daily. The second option uses two $22 \mathrm{~F}$ supercapacitators, giving a capacitance of $11 \mathrm{~F}$. This solution is the most expensive, but allows a node to operate for much longer without drawing from the primary capacity. Finally, we can also place a $150 \mathrm{mAh} \mathrm{Ni-Mh}$ rechargeable battery as primary buffer. Its energy capacity is superior to the $22 \mathrm{~F} \mathrm{su}-$ percapacitators by a factor of 17 , and it is also cheaper. Its disadvantage is that it tolerates a finite number of approximately 1000 charge cycles, unlike supercapacitators which have unlimited charge cycles. The secondary Li-Ion buffer is optional when using the Ni-Mh as primary buffer, allowing for a reduction of approximately $20 \%$ in component costs. Table 4 summarizes the three options for the primary energy buffer, and gives theoretical node primary buffer duration for three different duty cycles.

\subsection{Algorithm and Measurements}

We implemented a first driver in TinyOS following a strategy similar to Prometheus [14]. We tested the performance of our board with this algorithm, using three nodes: Node A had a $22 \mathrm{~F}$ supercap primary buffer, Node B had a $150 \mathrm{mAh}$ $\mathrm{Ni}-\mathrm{mH}$ battery, and Node $\mathrm{C}$ had a $1 \mathrm{~F}$ supercap. The algorithm constants were computed individually for each configuration. The three nodes were placed on a rooftop at EPFL for a three-month period starting in November 2005. Each node had a $10 \%$ radio duty cycle, representing a current draw of approximately $2 \mathrm{mAh}$. Nodes measured and transmitted $U_{\text {sec }}, U_{\text {prim }}, I_{\text {solar }}, I_{\text {charge }}$, and $I_{\text {supply }}$ every 10 seconds to a basestation in a neighboring office. All systems performed satisfactorily.

We focus now on the behavior of nodes A and B. We omit Node $\mathrm{C}$, which had a more monotonic and predictable behavior with very rapid charge/discharge cycles of its smaller primary buffer. Figure 6 shows the data collected in a three 


\begin{tabular}{|c|c|c|c|c|}
\hline $\begin{array}{l}\text { Duty } \\
\text { Cycle }\end{array}$ & $\begin{array}{l}2 \mathrm{x} 1 \mathrm{~F} \text { supercap. } \\
1.9 \mathrm{mWh} \\
1.6 \mathrm{~V}-5.5 \mathrm{~V}\end{array}$ & $\begin{array}{c}2 \times 22 \mathrm{~F} \text { supercap } \\
42.3 \mathrm{mWh} \\
1.6 \mathrm{~V}-5.5 \mathrm{~V}\end{array}$ & $\begin{array}{c}\text { 150mAh Ni-Mh bat. } \\
720 \mathrm{mWh} \\
5.3 \mathrm{~V}-4.4 \mathrm{~V}\end{array}$ & $\begin{array}{c}2000 \text { mAh Li-Ion bat. } \\
7400 \mathrm{mWh} \\
3.3 \mathrm{~V}-4.2 \mathrm{~V}\end{array}$ \\
\hline $\begin{array}{l}1 \% \\
10 \% \\
100 \%\end{array}$ & $\begin{array}{l}244 \text { minutes } \\
24 \text { minutes } \\
145 \text { seconds }\end{array}$ & $\begin{array}{c}90 \text { hours } \\
9 \text { hours } \\
54 \text { minutes }\end{array}$ & $\begin{array}{l}63.8 \text { days } \\
153 \text { hours } \\
15 \text { hours }\end{array}$ & $\begin{array}{l}656 \text { days } \\
65 \text { days } \\
6.5 \text { days }\end{array}$ \\
\hline
\end{tabular}

Table 4: Comparison of primary and secondary energy buffer options.
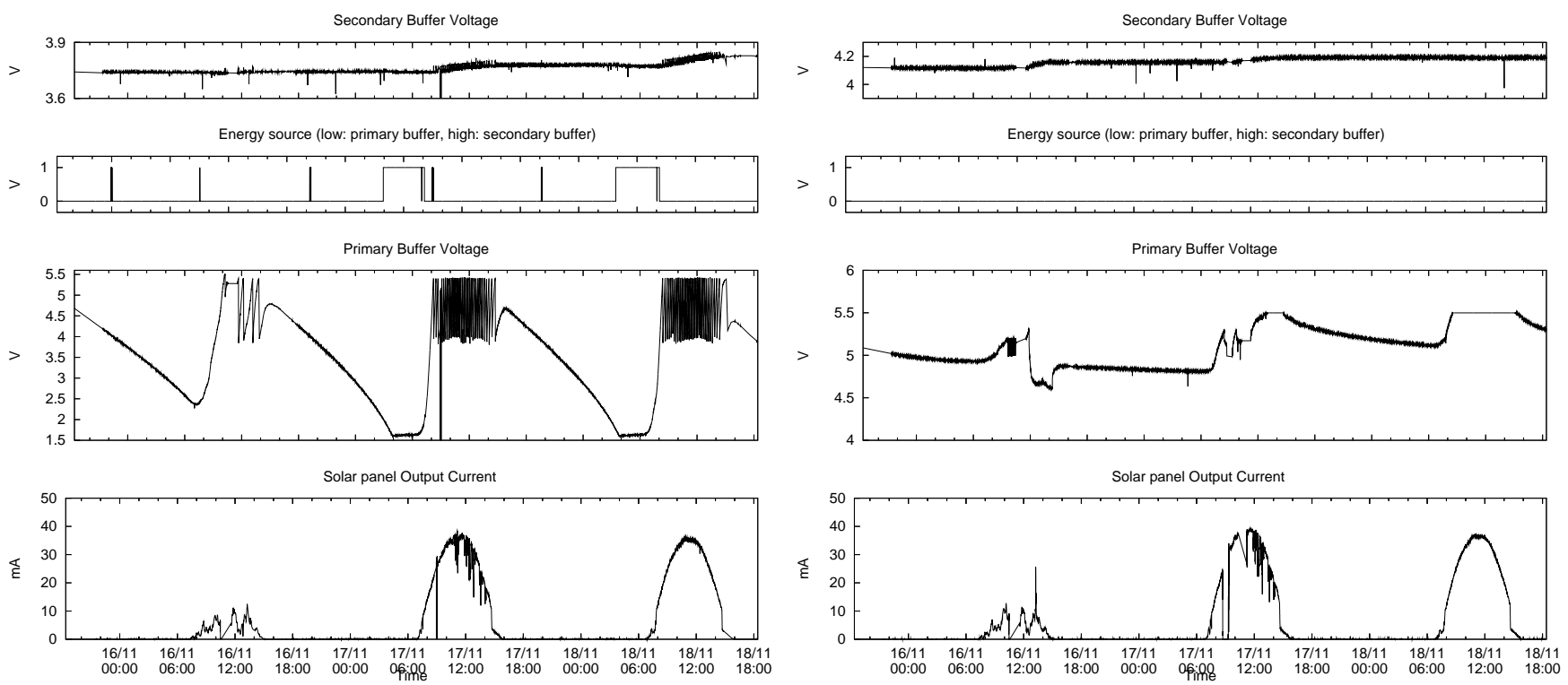

Figure 6: 72-hour energy traces. Left: node A (2 x 22F Supercapacitator). Right: node B (150 mAh Ni-mH battery).

day period. Day one was cloudy without any sunshine, and days two and three were clear and sunny (though with a fairly low winter sun). This is clearly observable in the bottom plot of solar panel output current. We can observe that for node $\mathrm{A}$, the Li-Ion accumulator had clear charge increases on days two and three, but not (or barely perceptible) on day one, whereas node B did increase the battery charge even on day one. This is due to the fact that node B's primary buffer is an order of magnitude larger than node A, and was still quite full going into day two. A small amount of light was sufficient to increase it to to $5.3 \mathrm{~V}$, at which point the driver initiated a charge cycle. Note that on day three, the voltage of node B's Li-Ion battery does not increase because it is fully charged at $4.2 \mathrm{~V}$. We now turn to the primary buffers. On node A, we can clearly see the daytime periodic charge cycles. Once the supercapacitators reached $5.4 \mathrm{~V}$, the charge switch opened into the Li-Ion battery until the supercapacitator ran down to $4 \mathrm{~V}$. These cycles are significantly slower on day one. We can see that on days two and three, node $\mathrm{A}$ does not quite make it through the night on the supercapacitator, and must switch to the secondary buffer for the last four hours of the night. This is not due to an insufficient capacity, but to simply because the capacitator was in the middle of a charge cycle when nightfall came. Without a control loop, the voltage of the capacitator will be randomly distributed between it's upper and lower operating points. Such a control loop should decrease the length and depth of the charge cycles toward the end of the day so as to start the night with a full primary buffer; we will implement one in the next iteration of the driver. Finally, we observe that node $\mathrm{B}$ never needed to use the secondary buffer in the entire period. In fact, with our $10 \%$ duty cycle node, the overall energy balance is clearly positive, since we can see that the secondary buffer is fully charged from the morning of day three (a Zehner diode prevents it from charging beyond $5.5 \mathrm{~V}$ ).

\section{CONCLUSION}

We have presented TinyNode, a new wireless sensor networking platform which comes with a rich set of hardware extensions for backhaul connectivity (ethernet, WLAN, and GPRS), mass storage, solar energy harvesting, and custom interfacing. The core module itself is a small, low power device, and uses a radio transceiver with range characteristics far beyond existing platforms. The node runs TinyOS with a complete radio stack and support for key subsystems such as network reprogramming. Several applications using TinyNodes are under development in the areas of environmental monitoring, precision agriculture, and parking management.

\section{ACKNOWLEDGEMENTS}

We thank Maxime Muller for his in-depth testing of the TinyOS radio stack, and Guillermo Barrenetxea for his help with the range tests. 


\section{REFERENCES}

[1] Alberto Cerpa, Jeremy Elson, Deborah Estrin, Lewis Girod, Michael Hamilton, and Jerry Zhao, "Habitat monitoring: Application driver for wireless communications technology," in 2001 ACM SIGCOMM Workshop on Data Communications in Latin America and the Caribbean, Costa Rica, 2001.

[2] Robert Szewczyk, Alan Mainwaring, Joseph Polastre, and David Culler, "An analysis of a large scale habitat monitoring application," in Proceedings of the Second ACM Conference on Embedded Networked Sensor Systems (SenSys), Baltimore, November 2004.

[3] Robert Adler, Phil Buonadonna, Jasmeet Chhabra, Mick Flanigan, Lakshman Krishnamurthy, Nandakishore Kushalnagar, Lama Nachman, and Mark Yarvis, "Design and deployment of industrial sensor networks: Experiences from the north sea and a semiconductor plant," in Proceedings of ACM Sensys, San Diego, USA, November 2005.

[4] Gilman Tolle, Joseph Polastre, Robert Szewczyk, Neil Turner, Kevin Tu, Phil Buonadonna, Stephen Burgess, David Gay, Wei Hong, Todd Dawson, and David Culler, "A macroscope in the redwoods," in Proceedings of ACM Sensys, San Diego, USA, November 2005.

[5] Jonathan W. Hui and David Culler, "The dynamic behavior of a data dissemination protocol for network programming at scale," in SenSys '04: Proceedings of the 2nd international conference on Embedded networked sensor systems, New York, NY, USA, 2004, pp. 81-94, ACM Press.

[6] Joseph Polastre, Robert Szewczyk, and David Culler, "Telos: Enabling ultra-low power wireless research," in The Fourth International Conference on Information Processing in Sensor Networks: Special track on Platform Tools and Design Methods for Network Embedded Sensors (IPSN/SPOTS), Los Angeles, California, Apr. 2005.
[7] V. Handziski, J. Polastre, J. H. Hauer, and C. Sharp, "Flexible hardware abstraction of the ti msp430 microcontroller in tinyos," in Proc. of SenSys '04: Proceedings of the 2nd international conference on Embedded networked sensor systems, Baltimore, MD, USA, Nov. 2004, pp. 277-278, ACM Press.

[8] Digi International, "Digi connect ME and digi connect Wi-ME," http://www.digi.com.

[9] V.Handziski, J. Polastre, J.-H. Hauer, C. Sharp, A. Wolisz, and D. Culler, "Flexible hardware abstraction for wireless sensor networks," in Proc. of 2nd European Workshop on Wireless Sensor Networks (EWSN 2005), Istanbul, Turkey, Feb. 2005.

[10] Joe Polastre, Jason Hill, and David Culler, "Versatile low power media access for wireless sensor networks," in Proceedings of ACM Sensys, Los Angeles, USA, April 2003.

[11] A. El-Hoiydi, J. Decotignie, and J. Hernandez, "Low power mac protocols for infrastructure wireless sensor networks," 2003.

[12] Linx Technologies, "868Mhz CW Series Antenna," http://www.linxtechnologies.com.

[13] Linx Technologies, "ANT-ELE-S01-011-ND Antenna," http://www.linxtechnologies.com.

[14] Xiaofan Jiang, Joseph Polastre, and David E. Culler, "Perpetual environmentally powered sensor networks.," in Proceedings of the Fourth International Symposium on Information Processing in Sensor Networks, IPSN 2005, April 25-27, 2005, UCLA, Los Angeles, California, USA. 2005, pp. 463-468, IEEE.

[15] Leclanché, "Lgc-1865," http://www.leclanche.ch/fr/produits/accumulateurs/li_ion.php.

[16] Cooper Industries, "Aerogel supercapacitators, b series," http://www.cooperet.com/library/products/PS-5102 B Series.pdf. 